\title{
Thermoregulatory Responses to Graded Exercise Differ among Sasang Types
}

\author{
Duong Duc Pham, ${ }^{1}$ Jeong Hoon Lee, ${ }^{1}$ Eun Seok Park, ${ }^{1}$ Hyun Sung Baek, ${ }^{1}$ Ga Yul Kim, \\ Young Boum Lee, ${ }^{1}$ BonCho Ku, ${ }^{2}$ Jong Yeol Kim, ${ }^{2}$ and Chae Hun Leem ${ }^{1}$ \\ ${ }^{1}$ Department of Physiology, Ulsan College of Medicine, 388-1 Poongnap-dong, Songpa-gu, Seoul 138-736, Republic of Korea \\ ${ }^{2}$ Korea Institute of Oriental Medicine, 461-24 Jeonmin-dong, Yuseong-gu, Daejeon 305-811, Republic of Korea
}

Correspondence should be addressed to Chae Hun Leem; leemch@gmail.com

Received 29 January 2015; Revised 6 May 2015; Accepted 11 May 2015

Academic Editor: I-Min Liu

Copyright (C) 2015 Duong Duc Pham et al. This is an open access article distributed under the Creative Commons Attribution License, which permits unrestricted use, distribution, and reproduction in any medium, provided the original work is properly cited.

\begin{abstract}
We compared sweat rate and variables such as workload $\left(W_{e}\right)$, metabolic heat production $\left(H_{\text {prod }}\right)$, and temperature increment load $\left(T_{\text {inc }}\right)$ across Sasang types. 304 apparently healthy participants aged 20-49 years with their Sasang type determined were enrolled. Local sweat rates on the chest $\left(\mathrm{LSR}_{\text {chest }}\right)$ and back $\left(\mathrm{LSR}_{\text {back }}\right)$ were measured using a perspiration meter during a maximum treadmill exercise test. Oxygen uptake was measured continuously using a breath-by-breath mode indirect calorimeter. The TaeEum (TE) type had a larger body size, a higher percent body fat, and a lower body area surface area (BSA) to body mass compared with the other Sasang types, particularly the SoEum (SE) type. The TE type tended to have a shorter exercise time to exhaustion and lower maximal oxygen uptake $\left(\mathrm{mL} \cdot \mathrm{kg}^{-1} \cdot \mathrm{min}^{-1}\right)$ than the other types. $\mathrm{LSR}_{\text {chest }}$ in TE types was greater than that of the SE and SoYang (SY) types in men, whereas $\mathrm{LSR}_{\text {back }}$ was higher in the TE type than that of the other types in women. After normalizing LSR for $W_{e}$, $H_{\text {prod }}, T_{\text {inc }}$, and BSA, this tendency still remained. Our findings suggest that the thermoregulatory response to graded exercise may differ across Sasang types such that the TE type was the most susceptible to heat stress.
\end{abstract}

\section{Introduction}

During exercise, $75 \%$ of metabolic energy is released as heat, causing body core temperature to rise. Thermoregulatory mechanisms maintain body temperature primarily through convection to the surrounding air and evaporation of sweat $[1,2]$. Anthropometric characteristics such as body size, body surface area- (BSA-) to-mass ratio (BSA/M), percent body fat, and subcutaneous adipose tissue affect thermoregulation during exercise [3-5].

Sasang constitutional medicine (SCM) is a form of traditional Korean medicine that focuses on the relationship between phenotypes and clinical features. According to this theory, humans can be classified into four constitutional types, TaeYang (TY), SoYang (SY), TaeEum (TE), and SoEum (SE), which differ in their physiological and psychological features, vulnerability to particular patterns of illnesses, and responses to external conditions including the thermoregulatory response [6,7]. According to SCM theory, sweat is an important sign of health states and determinant of human constitution. For instance, the TE type tends to sweat readily, and profuse sweating indicates a healthy state, whereas profuse sweating in the SE type, which is less prone to sweating, is the sign of an unhealthy state [6]. Several studies have shown that Sasang types have constitutionspecific physical, psychological, and genetic characteristics [8-11]. Several self-report studies of sweating have found that the TE and SY types tend to sweat more than the SE type under the same environmental conditions $[12,13]$. However, no studies have used objective and reliable methods to examine systematically thermoregulation and sweating capacity across Sasang types. We used an indirect calorimeter and hygrometer to objectively examine constitutionspecific sweat responses during graded exercise to investigate 
the effect of anthropometric features on thermoregulation among Sasang types.

\section{Methods}

2.1. Participants. A total of 550 apparently healthy volunteers (297 males and 253 females) between the ages of 20 and 49 years were recruited through advertisements at the Asan Medical Center in Seoul City, the capital of Republic of Korea, between 2009 and 2012. The study was approved by the Institutional Review Board of the Asan Medical Center and informed consent was obtained from all participants.

2.2. Classification of Sasang Type. We used the Sasang Constitution Analysis Tool (SCAT) developed by the Korea Institute of Oriental Medicine (KIOM) to determine Sasang type. The SCAT has been described in detail previously [14]. Briefly, the SCAT identifies Sasang type using a multinomial logistic regression analysis based on the integrated data of facial features (two-dimensional images), body shape (width and circumference), voice features, and questionnaire responses (personality traits and physiological symptoms). The accuracy of the SCAT has been reported to be higher than that of the Questionnaire for Sasang Constitution Classification II, the most commonly used diagnostic tool for Sasang typing $[14,15]$. Because the TY type is rare among Koreans, this version of the SCAT diagnosed only the TE, SE, and SY types.

2.3. Exclusion Criteria. Using the integrated data, the SCAT assessed the probability of each Sasang type for each participant (e.g., $\mathrm{TE}=0.53, \mathrm{SE}=0.40$, and $\mathrm{SY}=0.13$ ). $\mathrm{We}$ excluded individuals whose highest Sasang score was $<0.40$ and those in whom the difference between the highest and middle scores was $<0.05$ because their Sasang type was ambiguous $(n=237)$. The strict exclusion criteria were used to increase the reproducibility of the SCAT. The remaining 313 participants were classified according to their highest Sasang score, and their chest and back sweat rate were measured simultaneously during exercise. Further nine participants were excluded because they had unusual patterns of sweating (the increase in sweating was not steady and showed an abrupt increase and decrease during the early phase of exercise) or their oxygen consumption was not measured. Thus, the data of 304 participants (178 males and 126 females) were included in the analysis.

\subsection{Resting and Exercise Oxygen Consumption. Participants} were instructed to refrain from stimulants (smoking, alcohol, and coffee), heavy exercise, and eating a meal for $12 \mathrm{~h}$ prior to the experiment. Oxygen consumption $\left(\mathrm{VO}_{2}\right) 1 \mathrm{~min}$ before $\left(\mathrm{VO}_{2 \text { pre }}\right)$ and during exercise $\left(\mathrm{VO}_{2 \mathrm{ex}}\right)$ was measured using a breath-by-breath mode indirect calorimeter (Vmax ENCORE 29c-SensorMedics, VIASYS HealthCare, Yorba Linda, CA, USA). The $\mathrm{VO}_{2}$ measurement protocol has been described elsewhere [16]. Briefly, steady state $\mathrm{VO}_{2 \text { pre }}$ was measured while participants were seated on a chair, and $\mathrm{VO}_{2 \mathrm{ex}}$ was measured while participants ran on a treadmill following a standard Bruce protocol and were verbally encouraged to continue until exhaustion. $\mathrm{VO}_{2 \text { pre }}$ and $\mathrm{VO}_{2 \mathrm{ex}}$ were recorded at 20-s intervals and the average-per-minute measurements were used for further analyses. Furthermore, maximum oxygen consumption $\left(\mathrm{VO}_{2 \mathrm{max}}, \mathrm{mL} \cdot \mathrm{kg}^{-1} \cdot \mathrm{min}^{-1}\right)$, a measure of cardiorespiratory fitness, was recorded.

\subsection{Workload, Heat Production, and Estimated Temperature} Increment. Vertical displacement $\left(\mathrm{kg} \cdot \mathrm{m} \cdot \mathrm{min}^{-1}\right)$ was calculated by multiplying the percentage grade (\%) by speed $\left(\mathrm{m} \cdot \mathrm{min}^{-1}\right)$ and body weight $(\mathrm{kg})$. External workload $\left(W_{e}\right.$, Watt.min) was calculated as vertical displacement divided by 6.12 [17]. Total $W_{e}$ was the sum of $W_{e}$ per minute during exercise.

Metabolic energy expenditure $\left(M_{\mathrm{ee}}\right.$, Watt·min) for each minute before and during exercise was calculated from the respiratory quotient $(\mathrm{RQ})$ and $\mathrm{VO}_{2}$ using a modified equation described previously $[18,19]$, in which

$$
\begin{aligned}
& M_{\mathrm{ee}}(\text { Watt } \cdot \mathrm{min}) \\
& =\mathrm{VO}_{2}(\text { Litter }) \\
& \quad \times\left(\frac{(\mathrm{RQ}-0.7)}{0.3} \times 21.13+\frac{(1-\mathrm{RQ})}{0.3} \times 19.62\right) \\
& \quad \times 16.67 .
\end{aligned}
$$

Metabolic heat production $\left(H_{\text {prod }}\right.$, Watt·min) for each minute of exercise was calculated by subtracting $W_{e}$ from $M_{\text {ee }}$. The total $H_{\text {prod }}$ was the sum of $H_{\text {prod }}$ per minute during exercise. Metabolic efficiency (\%) was calculated as the percentage of $W_{e}$ to $M_{\mathrm{ee}}$.

Because 1 kilocalorie (69.78 Watt·min) of energy expenditure is required to raise 1 kilogram of body water by $1^{\circ} \mathrm{C}$ [17] and $M_{\mathrm{ee}}$ before exercise refers to the resting $M_{\mathrm{ee}}$ required to maintain normal body temperature, the temperature increment load $\left(T_{\text {inc }},{ }^{\circ} \mathrm{C}\right)$ for each is

$$
\begin{aligned}
& \left(T_{\text {inc }}\right)(\text { in degree Celsius }) \\
& =\frac{H_{\text {prod }}(\text { Watt } \cdot \min )-M_{\mathrm{ee}} \text { befor exercise }(\text { Watt } \cdot \min )}{69.78 \times \text { Body water }(\mathrm{kg})} .
\end{aligned}
$$

2.6. Local Sweat Rate Measurement. Local sweat rate (LSR) at the chest $\left(\mathrm{LSR}_{\text {chest }}\right)$ and back $\left(\mathrm{LSR}_{\text {back }}\right)$ was measured using a perspiration meter (SKN 2000, Nishizawa Electric Meters Manufacturing Co., Ltd, Nagano, Japan) that used a ventilated capsule to estimate LSR based on (i) the difference in vapor content between effluent and influent air passing through a given skin area and (ii) the air flow rate adjusted to the levels of sweating and varied in a range between 300 and $600 \mathrm{~mL} \cdot \mathrm{min}^{-1}[20]$. Two capsules were attached to the skin of the chest and back on the median line at the nipple level and using an adhesive band during the treadmill test. The treadmill exercise and sweat rate measurements were conducted at an ambient temperature of $23^{\circ} \mathrm{C} \pm 1^{\circ} \mathrm{C}$ and $60 \%$ humidity. Because the capsule covered a $1-\mathrm{cm}^{2}$ area of the skin, LSR was reported as mg per min per centimeter squared $\left(\mathrm{mg} \cdot \mathrm{cm}^{-2} \cdot \mathrm{min}^{-1}\right)$ at 20 -s intervals. $\mathrm{LSR}_{\text {chest }}$ and $\mathrm{LSR}_{\text {back }}$ were 
Table 1: Demographic characteristics, anthropometric indices, and maximal oxygen consumption according to Sasang type.

\begin{tabular}{|c|c|c|c|c|}
\hline & $\mathrm{TE}$ & SE & SY & $P$ \\
\hline \multicolumn{5}{|l|}{ Males } \\
\hline$n$ & 62 & 59 & 57 & \\
\hline Age (yrs) & $29.8(6.1)$ & $30.1(6.3)$ & $29.8(6.2)$ & 0.95 \\
\hline Weight (kg) & $82.6(9.4)^{\mathrm{a}, \mathrm{b}}$ & $62.8(6.4)^{\mathrm{c}}$ & $70.8(6.4)$ & $<0.001$ \\
\hline Height $(\mathrm{cm})$ & $174.9(5.5)^{\mathrm{a}}$ & $172.3(5.3)$ & $173.5(5.5)$ & 0.02 \\
\hline $\mathrm{BMI}\left(\mathrm{kg} \cdot \mathrm{m}^{-2}\right)$ & $27.0(2.6)^{\mathrm{a}, \mathrm{b}}$ & $21.2(1.8)^{\mathrm{c}}$ & $23.5(1.6)$ & $<0.001$ \\
\hline $\operatorname{BSA}\left(\mathrm{m}^{2}\right)$ & $1.98(0.13)^{\mathrm{a}, \mathrm{b}}$ & $1.74(0.10)^{\mathrm{c}}$ & $1.85(0.09)$ & $<0.001$ \\
\hline $\mathrm{BSA} / \mathrm{M}\left(\mathrm{cm}^{2} \cdot \mathrm{kg}^{-1}\right)$ & $241.1(13.0)^{\mathrm{a}, \mathrm{b}}$ & $278.9(14.9)^{\mathrm{C}}$ & $261.1(9.9)$ & $<0.001$ \\
\hline Fat-free mass (kg) & $61.6(5.9)^{\mathrm{a}, \mathrm{b}}$ & $52.2(4.7)^{\mathrm{C}}$ & $57.5(4.5)$ & $<0.001$ \\
\hline Body fat $(\mathrm{kg})$ & $21.1(6.5)^{\mathrm{a}, \mathrm{b}}$ & $10.6(3.4)^{\mathrm{c}}$ & $13.3(4.0)$ & $<0.001$ \\
\hline Body water (kg) & $45.1(4.3)^{\mathrm{a}, \mathrm{b}}$ & $38.4(3.5)^{\mathrm{C}}$ & $42.2(3.3)$ & $<0.001$ \\
\hline $\mathrm{VO}_{2 \max }\left(\mathrm{mL} \cdot \mathrm{kg}^{-1} \cdot \mathrm{min}^{-1}\right)$ & $532.7(97.1)^{\mathrm{a}, \mathrm{b}}$ & $739.2(121.0)^{\mathrm{c}}$ & $682.8(121.3)$ & $<0.001$ \\
\hline \multicolumn{5}{|l|}{ Females } \\
\hline$n$ & 46 & 40 & 40 & \\
\hline Age (yrs) & $31.9(6.7)$ & $30.6(7.1)$ & $28.1(5.2)$ & 0.02 \\
\hline Weight (kg) & $64.0(7.2)^{\mathrm{a}, \mathrm{b}}$ & $50.9(4.3)$ & $52.5(4.0)$ & $<0.001$ \\
\hline Height $(\mathrm{cm})$ & $159.9(5.1)$ & $161.3(5.2)$ & $159.0(5.7)$ & 0.18 \\
\hline $\mathrm{BMI}\left(\mathrm{kg} \cdot \mathrm{m}^{-2}\right)$ & $25.1(2.7)^{\mathrm{a}, \mathrm{b}}$ & $19.6(1.5)^{\mathrm{c}}$ & $20.8(1.6)$ & $<0.001$ \\
\hline $\operatorname{BSA}\left(\mathrm{m}^{2}\right)$ & $1.66(0.10)^{\mathrm{a}, \mathrm{b}}$ & $1.52(0.08)$ & $1.53(0.08)$ & $<0.001$ \\
\hline $\mathrm{BSA} / \mathrm{M}\left(\mathrm{cm}^{2} \cdot \mathrm{kg}^{-1}\right)$ & $261.6(15.5)^{\mathrm{a}, \mathrm{b}}$ & $299.3(12.1)^{\mathrm{c}}$ & $291.1(11.0)$ & \\
\hline Fat-free mass (kg) & $42.5(4.0)^{\mathrm{a}, \mathrm{b}}$ & $37.9(3.6)$ & $38.3(3.7)$ & $<0.001$ \\
\hline Body fat (kg) & $21.5(5.2)^{\mathrm{a}, \mathrm{b}}$ & $13.1(3.1)$ & $14.2(2.8)$ & $<0.001$ \\
\hline Body water (kg) & $31.1(3.0)^{\mathrm{a}, \mathrm{b}}$ & $27.8(2.7)$ & $28.1(2.7)$ & $<0.001$ \\
\hline $\mathrm{VO}_{2 \max }\left(\mathrm{mL} \cdot \mathrm{kg}^{-1} \cdot \min ^{-1}\right)$ & $546.1(114.5)^{\mathrm{a}, \mathrm{b}}$ & $758.2(136.8)$ & $716.5(129.2)$ & $<0.001$ \\
\hline
\end{tabular}

Data are expressed as means (SD). BMI, body mass index; BSA, body surface area; BSA/M, body surface area per body weight; $\mathrm{VO}_{2 \max }, \mathrm{maximal}$ oxygen consumption during the treadmill exercise. Sasang typology: TE, Taeum type; SE, Soeum type; SY, Soyang type. $P$ values were calculated using one-way analysis of variance. Tukey's HSD post hoc test: ${ }^{a} \mathrm{TE}$ differed from SE; ${ }^{\mathrm{b}} \mathrm{TE}$ differed from SY; ${ }^{\mathrm{c}} \mathrm{SE}$ differed from SY.

measured continuously during exercise, and the average-perminute values were used in the analysis. Whole-body sweat rate (WSR) at the chest ( $\left.\mathrm{WSR}_{\text {chest }}\right)$ and back $\left(\mathrm{WSR}_{\text {back }}\right)$ was calculated as $\mathrm{LSR}_{\text {chest }}$ and $\mathrm{LSR}_{\text {back }}$ multiplied by BSA $\left(\mathrm{cm}^{2}\right)$, respectively. In a further analysis, sweat-induced factors were normalized by dividing LSR and WSR by $W_{e}, H_{\text {prod }}$, and $T_{\text {inc }}$. The increment in sweat rate (SR) per each minute of exercise was calculated by subtracting the SR measured at the previous minute. The analysis was performed for LSR and WSR.

2.7. Body Composition Measurement. Body fat mass (BFM), fat-free mass (FFM), and body water were measured by bioimpedance analysis using the InBody 720 (Biospace, Seoul, Korea). The device uses a segmental multifrequency impedance technique, and its body composition measurements are comparable to those obtained using dual-energy Xray absorptiometry (DXA), the standard body composition analyzer [21]. BSA was calculated using the formula of D. Du Bois and E. F. Du Bois [22]. Body weight and height were measured using a digital scale.

2.8. Data Analysis. All statistical analyses were conducted according to sex using R 3.1.2 for Windows. One-way analysis of variance (ANOVA) was used to evaluate differences in the demogr ic characteristics, body composition, cardiorespiratory fitness, exercise time, total $W_{e}$, total $M_{\text {ee }}$, and metabolic efficiency across Sasang types. Tukey's HSD test was used for post hoc comparisons.

The time-dependent analysis could not be performed using a repeated measures test because the exercise times differed among participants; thus, the Kruskal-Wallis rank sum test was used to assess differences in the mean rank of $W_{e}, M_{\mathrm{ee}}, T_{\mathrm{inc}}$, and SR values for each minute of exercise. Significant differences were followed up using the Nemenyi test for post hoc pairwise multiple comparisons of ranked data using package PMCMR [23]. $P$ values $<0.05$ were deemed to indicate statistical significance.

\section{Results}

3.1. Demographic Characteristics, Anthropometric Indices, and $V_{2 \max }$. There was no age difference among Sasang types in men, whereas the TE women were older than SY women $(P<$ $0.05)$. Conversely, height did not differ among Sasang types in females, whereas TE type males were taller than those in the $\mathrm{SE}$ group. The body size of the TE type males was larger than 

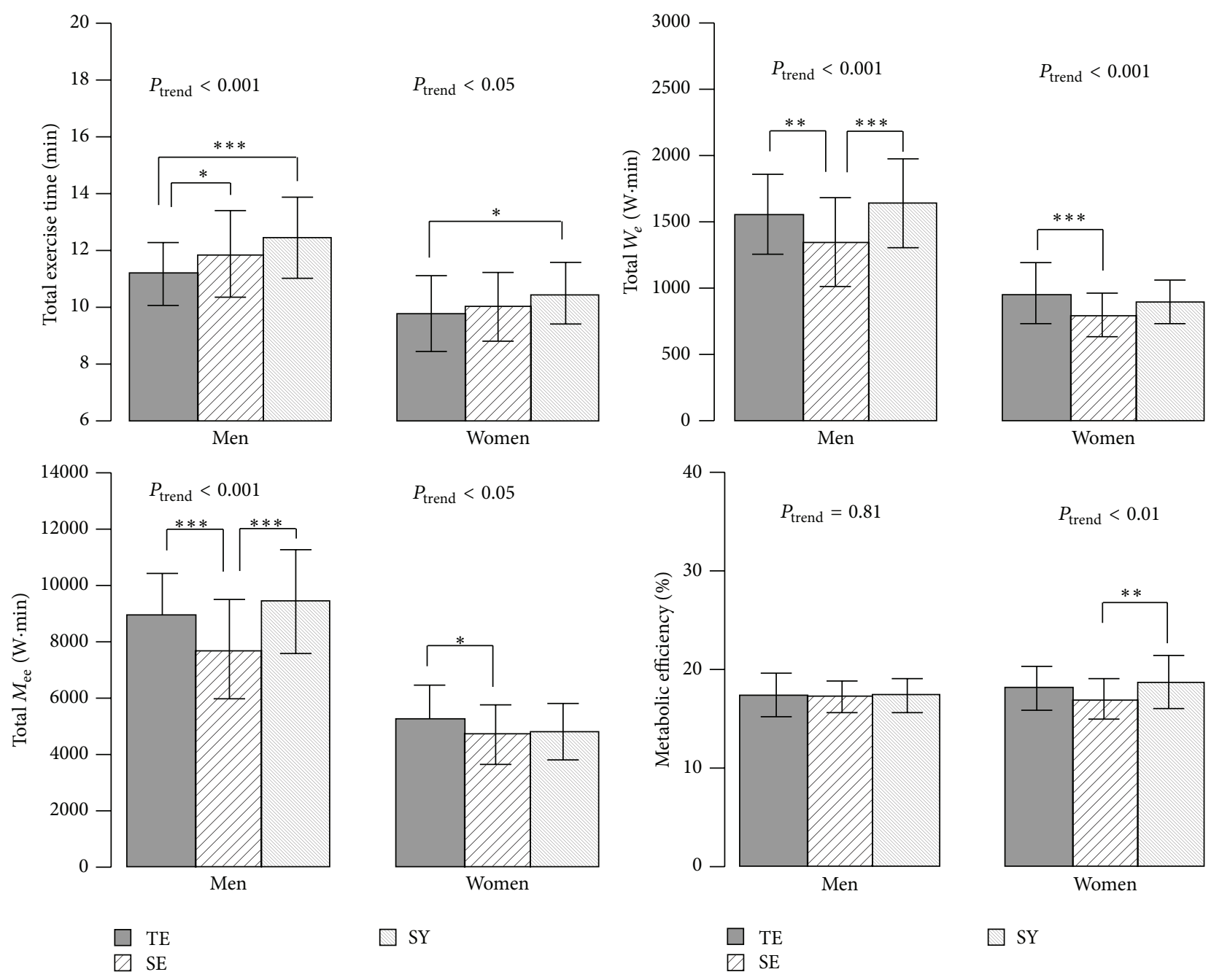

Figure 1: Total exercise time, external workload $\left(W_{e}\right)$, metabolic energy expenditure $\left(M_{\mathrm{ee}}\right)$, and metabolic efficiency $\left(W_{e}\right.$ divided by $\left.M_{\mathrm{ee}} \%\right)$ across Sasang types by gender. Data are presented as mean (SD). Sasang types include TaeEum type, SoEum type, and SoYang type. $P_{\text {trend }}$ calculated by one-way ANOVA test. Tukey's HSD post hoc test: ${ }^{*} P<0.05,{ }^{* *} P<0.01$, and ${ }^{* * *} P<0.001$.

that of the other types and, as a consequence, body weight, BMI, BFM, FFM, BSA, and body water content were higher. The body size-linked values of the SE group were lower than those of the TE and SY types. Similarly, TE type females had a larger body size and higher body weight, BMI, BFM, FFM, BSA, and body water content than those in the SY and SE groups, although the body size-linked values did not differ between the SE and SY types.

$\mathrm{VO}_{2 \max }$ was highest among SY type males and lowest among those in the TE group, whereas $\mathrm{VO}_{2 \max }$ was comparable between females in the SY and SE types and lowest in the TE group (Table 1).

3.2. Exercise Time, $W_{e}, M_{e e}$, and $T_{i n c}$. Despite a similar total $W_{e}$ (post hoc test, $P=0.34$ in males and $P=0.21$ in females) and an equal total $M_{\mathrm{ee}}$ in males (post hoc test $P=0.81$ ), the exercise times of TE type males and females were shorter than those of the SY type males $(11.1 \pm 1.1$ versus $12.4 \pm$
1.4 min, resp.; $P<0.001)$ and females $(9.8 \pm 1.3$ versus $10.4 \pm$ $1.1 \mathrm{~min}$, resp.; $P<0.05)$. In contrast, the comparison between TE and SE type males revealed that SE males had a lower total $W_{e}$ (post hoc test, $P<0.01$ ) and lower total $M_{\text {ee }}$ (post hoc test, $P<0.05$ ) over a longer exercise period (post hoc test $P<0.05)$ than did TE males. Exercise time did not differ between the TE and SE type females, although the TE type females had a higher $W_{e}$ than did SE females. The only difference in metabolic efficiency across Sasang types was that observed between SE and SY type females (Figure 1).

Figure 2 shows that $W_{e}, M_{\mathrm{ee}}$, metabolic efficiency, and $T_{\text {inc }}$ increased during exercise. In males, the $W_{e}$ and $M_{\mathrm{ee}}$ were significantly different among Sasang types at each exercise minute such that the values were highest in the TE and lowest in the SE type individuals. No differences in metabolic efficiency were found among Sasang types. TE type males had a higher $T_{\text {inc }}$ between 2 and 10 min of exercise than did those in the SE group. In females, $W_{e}$ and $M_{\mathrm{ee}}$ were higher in the TE type than in the SY and SE type individuals; however, 

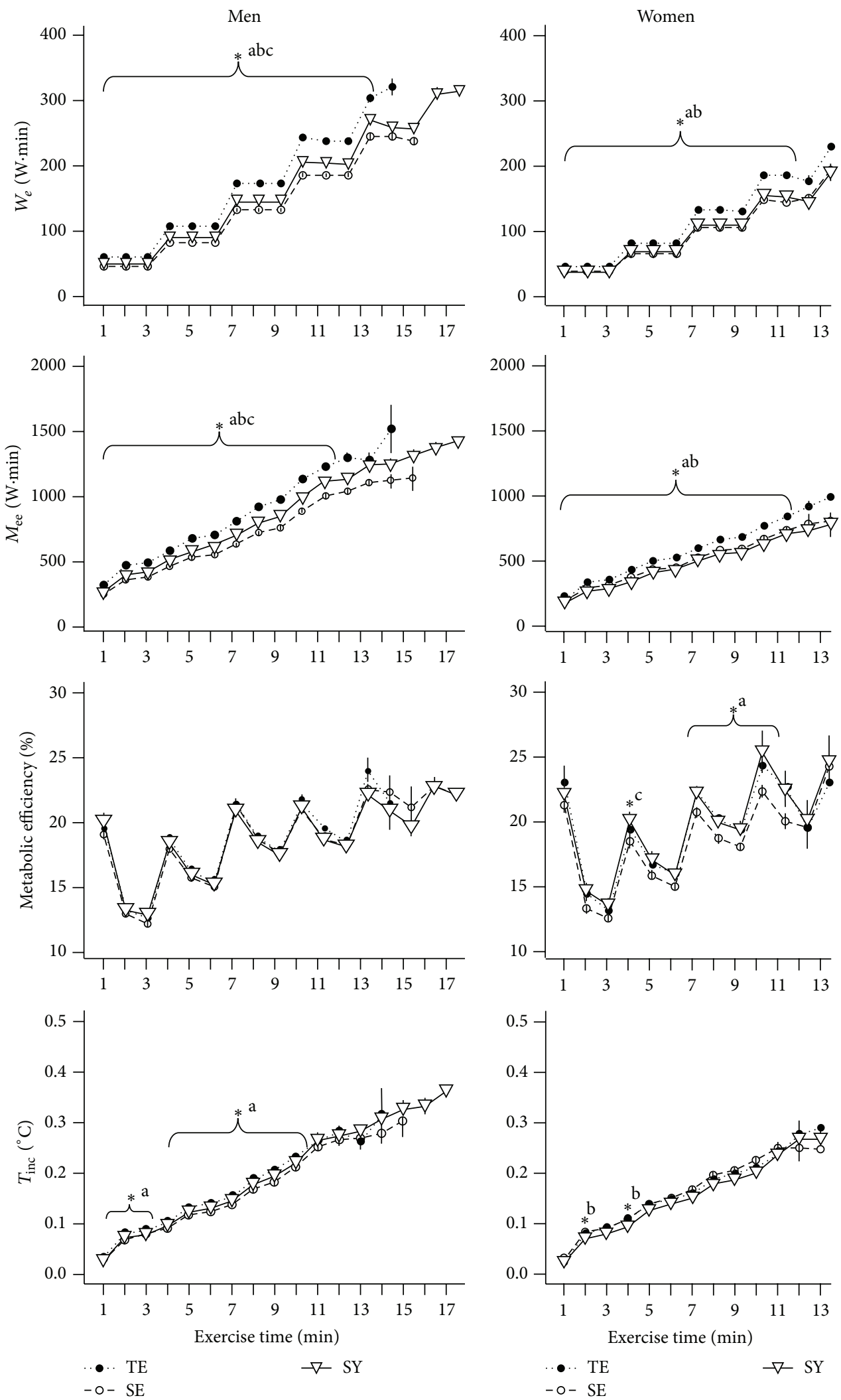

FIGURE 2: External workload $\left(W_{e}\right)$, metabolic energy expenditure $\left(M_{\mathrm{ee}}\right)$, metabolic efficiency ( $W_{e}$ to $M_{\mathrm{e} e} \%$ ), and temperature increment load $\left(T_{\text {inc }}\right)$ across Sasang types by gender. Data are presented as mean (SEM). TE, TaeEum type; SE, SoEum type; SY, SoYang type. * Significant difference between groups at each time point $(P<0.05)$ by Kruskal-Wallis rank sum test. Nemenyi's post hoc test: ${ }^{\mathrm{a}} \mathrm{TE}$ differs from SE; ${ }^{\mathrm{b}} \mathrm{TE}$ differs from SY; ${ }^{\mathrm{C} E}$ differs from SY. 

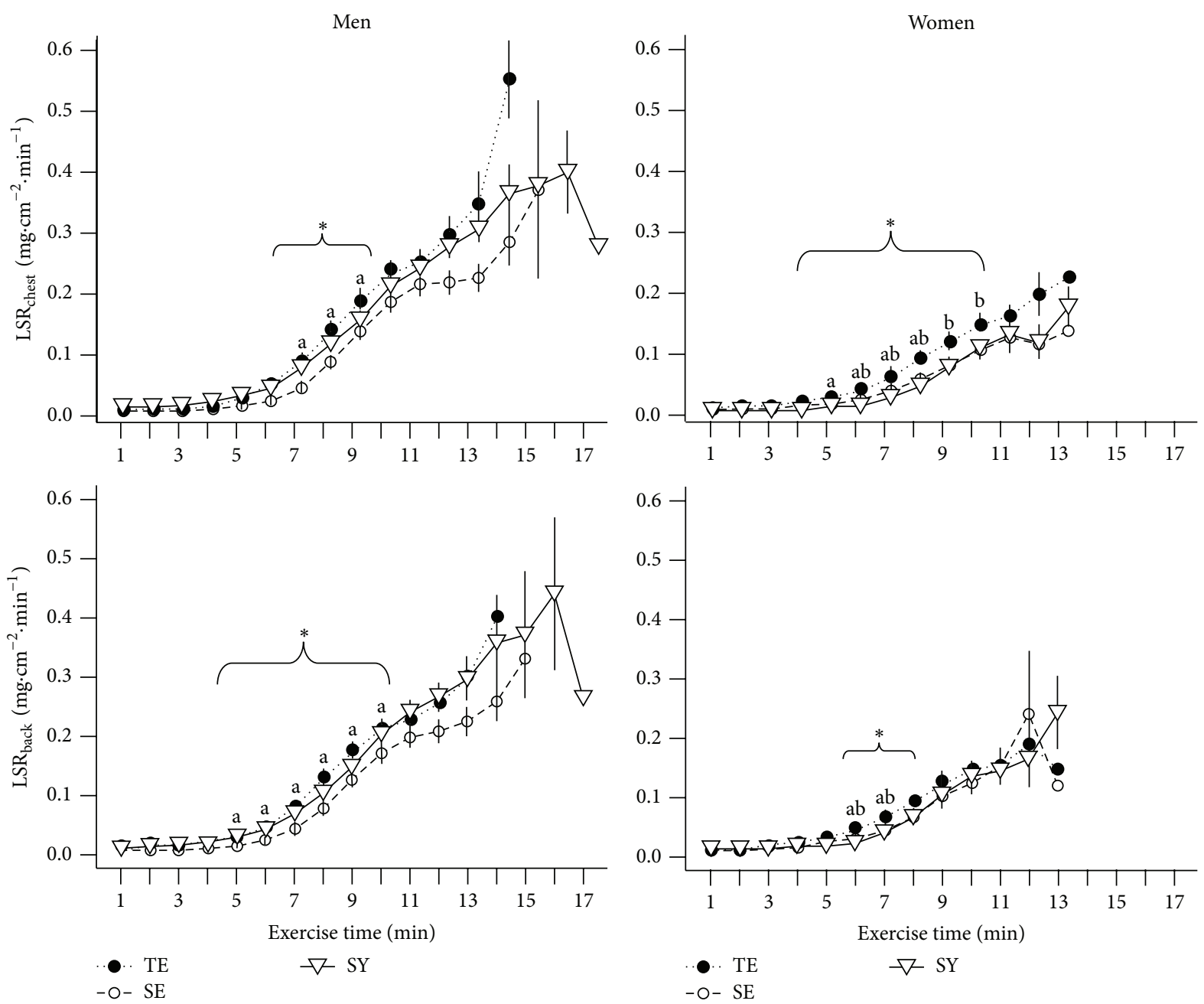

FIgURE 3: Local sweat rate measured on the chest $\left(\mathrm{LSR}_{\text {chest }}\right)$ and on the back $\left(\mathrm{LSR}_{\text {back }}\right)$ across Sasang types by gender. Data are presented as mean (SEM). TE, TaeEum type; SE, SoEum type; SY, SoYang type. ${ }^{*}$ Significant difference between groups at each time point $(P<0.05)$ by Kruskal-Wallis rank sum test. Nemenyi's post hoc test: ${ }^{a}$ TE differs from SE; ${ }^{\text {b } T E ~ d i f f e r s ~ f r o m ~ S Y ; ~}{ }^{\mathrm{c}} \mathrm{SE}$ differs from SY.

these values did not differ between the SY and SE types. TE type females tended to have higher metabolic efficiency than those in the SE group; however, the difference was statistically significant at only a few exercise time points. With the exception of the early stage of exercise, the $T_{\text {inc }}$ did not tend to differ among Sasang types in females.

3.3. Local and Whole-Body Sweat Rate during Exercise. Figure 3 shows $L_{S R}$ chest and $L_{S R}$ back for each minute during the treadmill exercise across Sasang types according to sex. We found no difference in LSR values during the early stage of exercise; however, $\mathrm{LSR}_{\text {chest }}$ and $\mathrm{LSR}_{\text {back }}$ were higher in the TE than in the SY and SE types after 7 and $5 \mathrm{~min}$, respectively, in males and after 5 and $6 \mathrm{~min}$, respectively, in females. The LSR differences between Sasang types were ambiguous during the late stage of exercise because few TE and SE individuals were able to continue exercise for $17 \mathrm{~min}$. We found a similar pattern in WSR: there were no differences in
$\mathrm{WSR}_{\text {chest }}$ and $\mathrm{WSR}_{\text {back }}$ among Sasang types during the early stage of exercise, with the exception that TE females had a

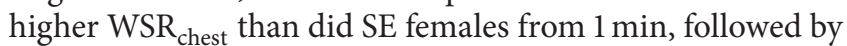
a significant increase in $\mathrm{WSR}_{\text {chest }}$ and $\mathrm{WSR}_{\text {back }}$ in TE type individuals compared with those in the SY and the SE group in particular, during the middle stage (Figure 4).

3.4. Sweat Rate Normalized to Sweat-Induced Factors during Exercise. We found no differences among Sasang types in WSR $_{\text {chest }}$ normalized to $W_{e}$ and $H_{\text {prod }}$ in males (Figures 5(a) and $5(\mathrm{e}))$ and in $\mathrm{WSR}_{\text {back }}$ normalized to $W_{e}$ and $H_{\text {prod }}$ in females (Figures 5(d) and 5(h)). However, the TE type tended to show a greater increase in $\mathrm{WSR}_{\text {back }}$ normalized to $W_{e}$ and $H_{\text {prod }}$ in males and $\mathrm{WSR}_{\text {chest }}$ normalized to $W_{e}$ and $H_{\text {prod }}$ in females (Figures 5(c), 5(g), 5(b), and 5(f), resp.). WSR chest and WSR $_{\text {back }}$ normalized to $T_{\text {inc }}$ were higher in TE type than in the SY, and the SE individuals in particular, during the middle stage of exercise (Figures 5(i), 5(j), 5(k), and 5(l)). 

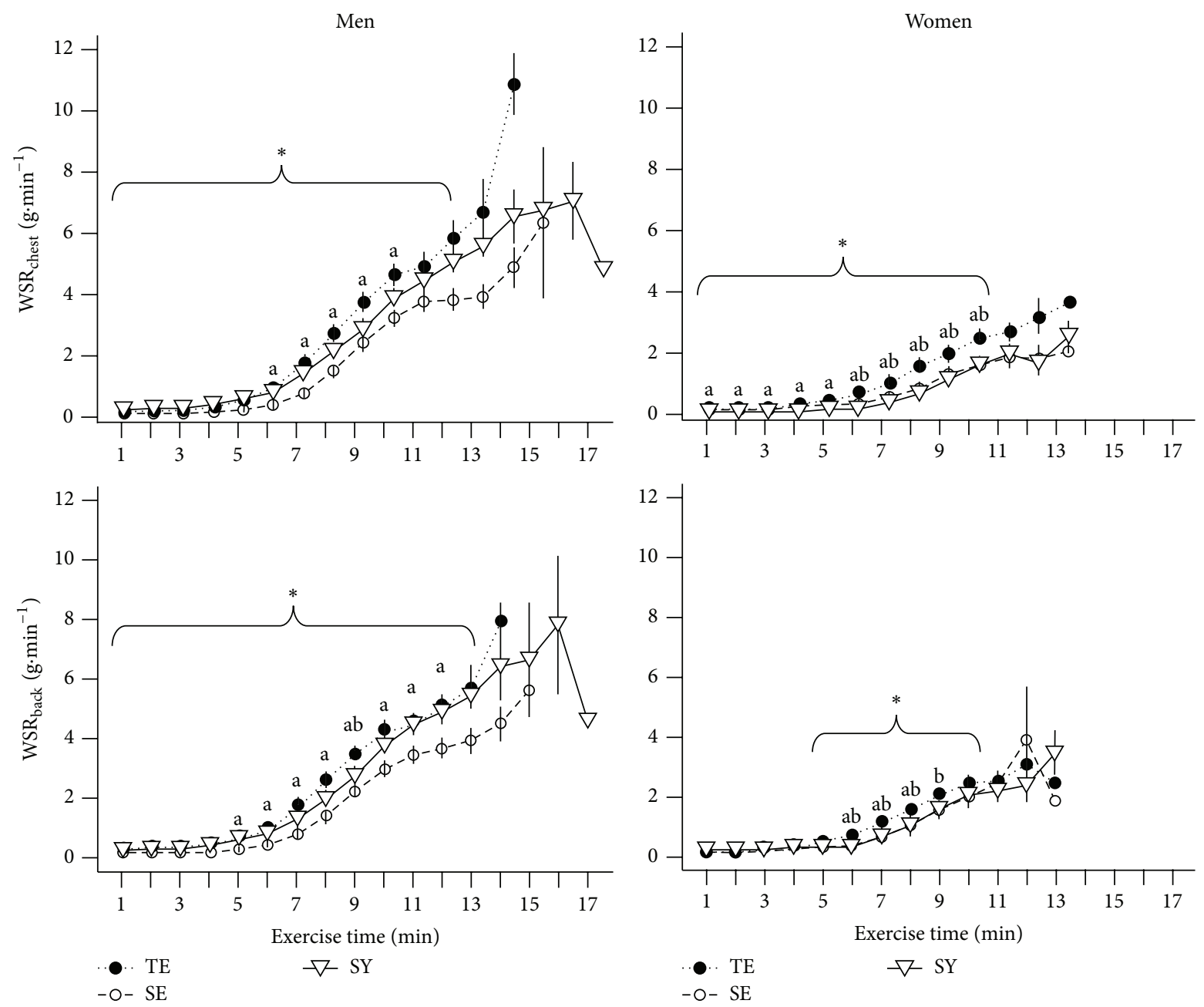

FIGURE 4: Whole-body sweat rate estimated by multiplying body surface are with $\mathrm{LSR}_{\text {chest }}\left(\mathrm{WSR}_{\text {chest }}\right)$ and with $\mathrm{LSR}_{\text {back }}\left(\mathrm{WSR}_{\text {back }}\right)$ across Sasang types by gender. Data are presented as mean (SEM). TE, TaeEum type; SE, SoEum type; SY, SoYang type. ${ }^{*}$ Significant difference between groups at each time point $(P<0.05)$ by Kruskal-Wallis rank sum test. Nemenyi's post hoc test: ${ }^{\mathrm{a}} \mathrm{TE}$ differs from SE; ${ }^{\mathrm{b}} \mathrm{TE}$ differs from SY; ${ }^{\mathrm{c}} \mathrm{SE}$ differs from SY.

Analysis of the per-min increment in WSR normalized to $W_{e}, H_{\text {prod }}$, and $T_{\text {inc }}$ revealed that, compared with the other types, TE males showed a greater increase in $\mathrm{WSR}_{\text {back }}$ normalized to $W_{e}$ and $H_{\text {prod }}$ at 6 and 7 min of exercise, whereas in females, WSR $\mathrm{Whest}_{\text {and }}$ WSR $\mathrm{back}_{\text {bormalized to }} W_{e}$ and $H_{\text {prod }}$ were higher in the TE than other Sasang types. Normalization of WSR to $T_{\text {inc }}$ revealed a consistent elevation of SR in the TE type individuals at the 6 and 7 min of exercise (see Figure S1 available online at http://dx.doi.org/10.1155/2015/879272). Furthermore, this pattern was observed in the $\mathrm{LSR}_{\text {chest }}$ and $\mathrm{LSR}_{\text {back }}$ data (Figures S2 and S3).

\section{Discussion}

Our study is the first to investigate the SR produced by graded exercise in Sasang typology. Our primary finding was that the SR was highest among TE and lowest among SE participants during the middle stage of graded exercise, and this difference persisted when SR was normalized to sweat-induced factors such as $W_{e}, H_{\text {prod }}$, and $T_{\text {inc }}$. We also found that the $T_{\text {inc }}$ was higher in the TE type during exercise, particularly in men, and that the TE type had a shorter total exercise time to exhaustion than other Sasang types.

Individual variations in anthropometric characteristics, such as BSA, BSA/M, adipose tissue thickness, and intensity of the exercise, have been shown to affect the thermoregulatory response. Evidence indicates that smaller body size, regardless of training status, can endure a greater maximal physical workload $[24,25]$. Yokota et al. $[26,27]$ found that core temperature during physically related heat stress was likely to be higher in males and females with high percent body fat than in lean individuals. This may be because smaller individuals have a high BSA/M, which is a distinct thermal advantage for heat loss, and the homeostatic imbalance caused by heat retention during exercise is controlled more efficiently. The significant amount of heat generated by exercise may be a crucial factor in the reluctance of heavier 


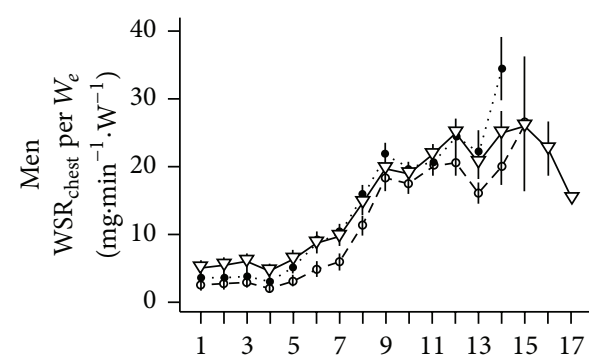

(a)

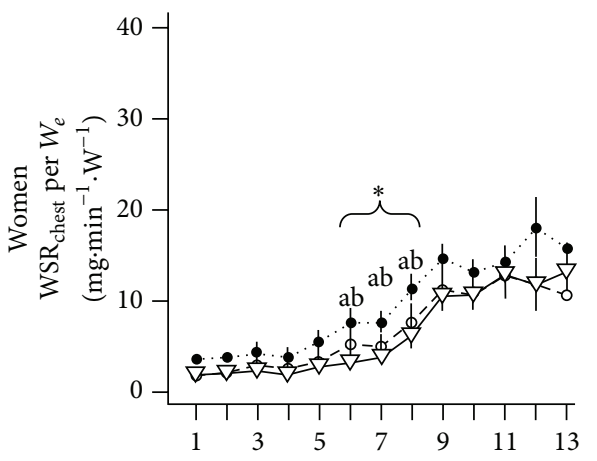

(b)

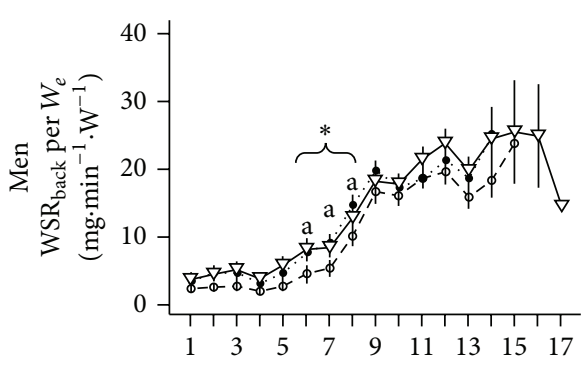

(c)

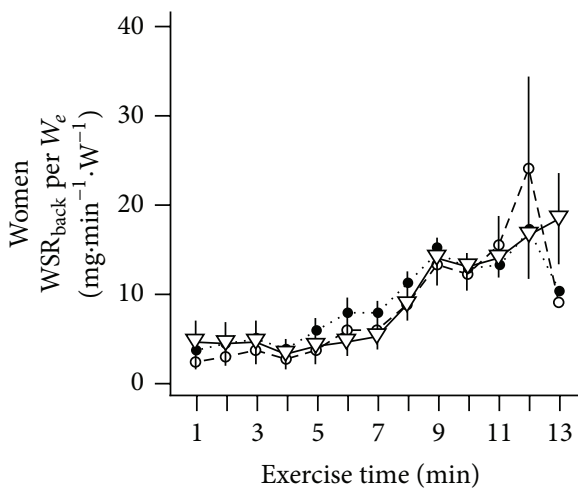

- $\mathrm{TE}$

$-\nabla$ SY

(d)

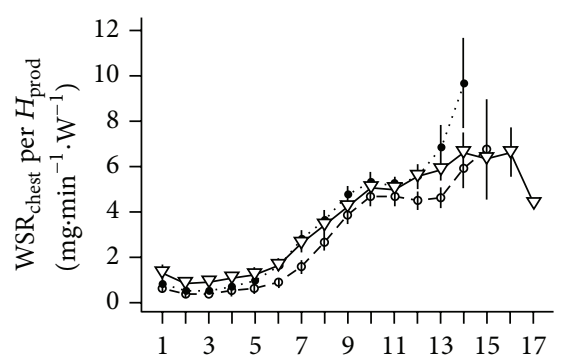

(e)

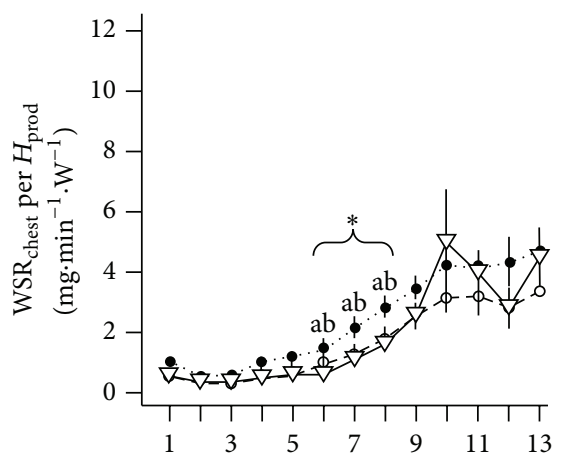

(f)

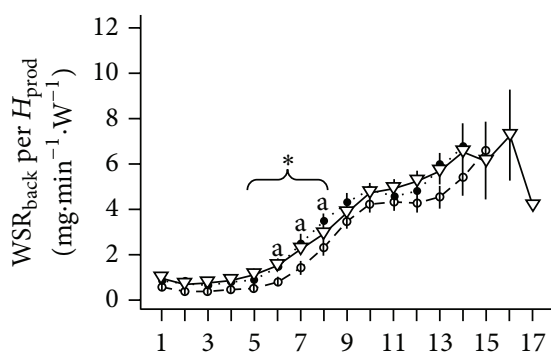

(g)

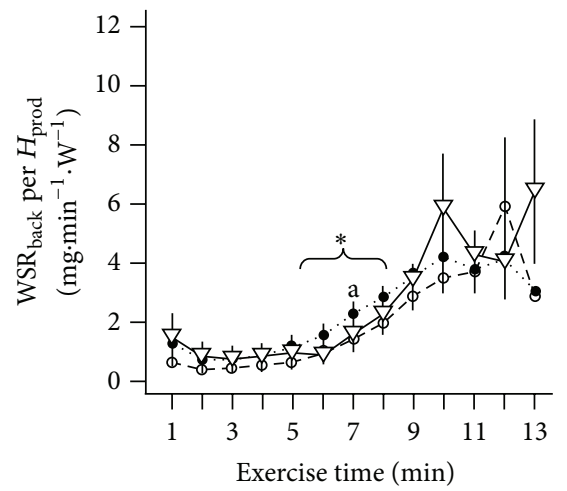

-. TE
$-\mathrm{O}-\mathrm{SE}$

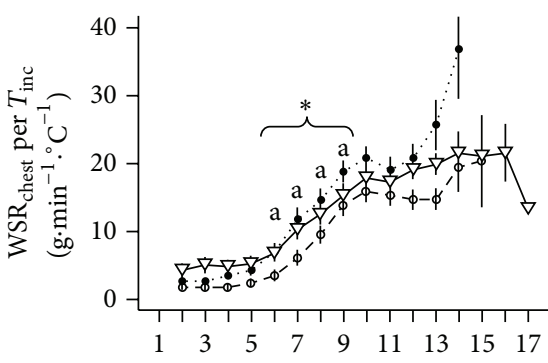

(i)

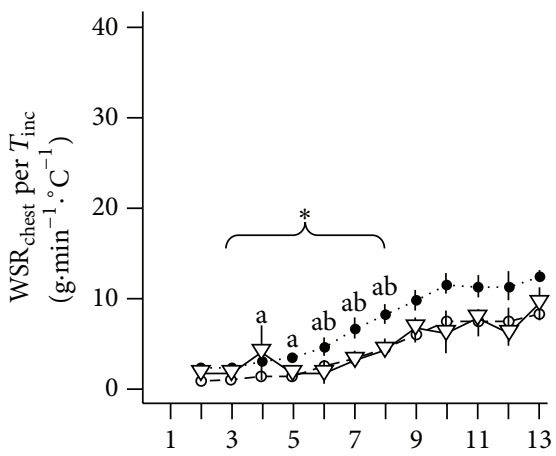

(j)

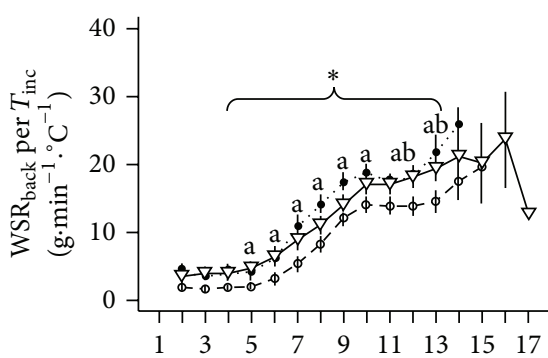

(k)

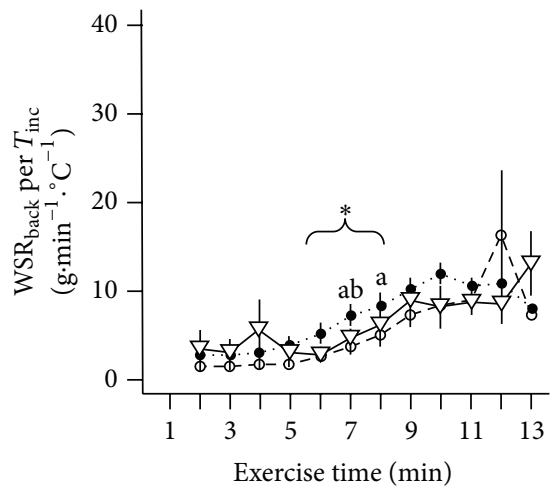

-. TE -O- SE

(l)

FIGURE 5: Whole-body sweat rate estimated from $\mathrm{LSR}_{\text {chest }}$ and $\mathrm{LSR}_{\text {back }}$ divided by external workload (WSR $\mathrm{Whest}_{\text {per }} W_{e}$ and $\mathrm{WSR}_{\mathrm{back}}$ per $\left.W_{e}\right)$, by metabolic heat production $\left(\mathrm{WSR}_{\text {chest }}\right.$ per $H_{\text {prod }}$ and $\mathrm{WSR}_{\text {back }}$ per $H_{\text {prod }}$ ), and by temperature increment $\left(\mathrm{WSR}_{\text {chest }}\right.$ per $T_{\text {inc }}$ and WSR $\mathrm{Wack}_{\text {b }}$ per $\left.T_{\text {inc }}\right)$ across Sasang types by gender. Data are presented as mean (SEM). TE, TaeEum type; SE, SoEum type; SY, SoYang type. * Significant difference between groups at each time point $(P<0.05)$ by Kruskal-Wallis rank sum test. Nemenyi’s post hoc test: ${ }^{\mathrm{a}} \mathrm{TE}$ differs from SE; ${ }^{\mathrm{b}} \mathrm{TE}$ differs from SY; ${ }^{c}$ SE differs from SY. 
individuals to engage in prolonged physical activities [28, 29]. The anthropometric characteristics of the Sasang types in our study (i.e., larger body size, higher percent of fat mass, and a lower BSA/M in the TE compared with the other types and small body size, low fat mass percentage, and high BSA/M in the SE individuals) were comparable with those reported previously $[16,30]$. Although we did not measure the change in body temperature during exercise, the estimated $T_{\text {inc }}$ based on oxygen consumption showed the greatest increase in the TE type participants. Increase in $T_{\text {inc }}$ during graded exercise may induce a higher $\mathrm{SR}$, a lower $\mathrm{VO}_{2 \max }$, a proxy for physical fitness, and a shorter time to exhaustion in the TE type.

Because the TE type participants had a higher BMI and body fat percentage than did the SY and SE types, the subcutaneous adipose tissue, a layer of impeding thermal convection, may have been thicker in the TE type individuals. Moreover, the relative surface for heat convection, the $\mathrm{BSA} / \mathrm{M}$, was smaller in TE type participants; thus, heat loss was conducted primarily through evaporation by sweating rather than convection via the skin-environment thermal gradient. Recently, Kim et al. [31] reported that the TE type subjects had higher viscoelasticity and lower elasticity hysteresis at the forearm than did the SE type individuals. Further studies are needed to investigate the constitutionspecific differences in convection and radiation activity and skin blood flow during exercise.

Several studies have shown that sweating sensitivity in individuals native to tropical regions, who have smaller, thinner bodies than those native to temperate climate zones, was relatively lower than that of temporal region natives $[32,33]$. This physiological trait may be a crucial factor in the protection of tropical natives from heat stress, particularly during prolonged physical activity. Interestingly, the response to heat stress in short-term heat adapted individuals is related to an increase in the amount of sweat produced, whereas long-term heat acclimation was associated with the economical use of body fluid [30]. Thus, it is reasonable to suggest that the potential differences in thermoregulatory responses among Sasang typologies may be due to inherited long-term heat acclimation in which sweating sensitivity may play an important role. We did not examine sweat gland function in the present study and further investigation is warranted.

Different body sizes among the Sasang types were associated with differences in $W_{e}$ and $H_{\text {prod }}$ as skin surface areas differed. In theory, participants who performed at the same work intensity produced the same amount of heat and released the same total amount of sweat, whilst those with a higher BSA have a lower LSR despite producing the same total amount of sweat. Thus, Cramer et al. [18] proposed that LSR be compared under the same work intensity/heat production per square of BSA. Our analyses of the absolute LSR, LSR normalized to sweat-induce factors $\left(W_{e}, H_{\text {prod }}\right.$, and $\left.T_{\text {inc }}\right)$, and WSR all showed that the graded exercise-induced increase in SR was greater in TE type participants than in those of the other Sasang types.

Our findings should be interpreted in the context of several limitations. We assessed the LSR, which does not reflect whole-body evaporation. Furthermore, we consider this to be a pilot study because we examined only the macrophenomenon related to the sweating response and did not investigate the pattern of relevant variables such as body core and skin temperature and the distribution and activation of sweating glands. Thus, we were unable to provide a mechanistic explanation for the distinct sweating response patterns we observed among the constitutions.

In conclusion, our findings indicate that the thermoregulatory response to graded exercise may differ across Sasang types such that the TE type was the most susceptible to heat stress induced by prolonged exercise. Several TE-specific traits including high body fat mass, low BSA-to-mass ratio, thicker subcutaneous adipose tissue, and lower aerobic fitness may cause earlier exhaustion, earlier sweating onset, and a greater increase in sweat production during the treadmill exercise in TE type individuals compared with the other Sasang types.

\section{Conflict of Interests}

The authors declare that there is no conflict of interests regarding the publication of this paper.

\section{Authors' Contribution}

Chae Hun Leem and Jong Yeol Kim designed and supervised the study and edited the paper as corresponding authors. Duong Duc Pham designed the methods, analyzed the data, interpreted the results, and wrote the paper. BonCho $\mathrm{Ku}$ coanalyzed the data, interpreted the results, and edited the paper. Jeong Hoon Lee, Eun Seok Park, Hyun Sung Baek, Ga Yul Kim, and Young Boum Lee collected data.

\section{Acknowledgment}

This work was supported by the Bio and Medical Technology Development Program of the National Research Foundation of Korea (NRF) funded by the Ministry of Science, ICT and Future Planning (nos. 2006-2005173 and 2012-0009829).

\section{References}

[1] A. C. Guyton and J. E. Hall, Textbook of Medical Physiology, Saunders, Philadelphia, Pa, USA, 1996.

[2] T. Rowland, "Thermoregulation during exercise in the heat in children: old concepts revisited," Journal of Applied Physiology, vol. 105, no. 2, pp. 718-724, 2008.

[3] J. D. Coso, N. Hamouti, J. F. Ortega, V. E. Fernández-Elías, and R. Mora-Rodríguez, "Relevance of individual characteristics for thermoregulation during exercise in a hot-dry environment," European Journal of Applied Physiology, vol. 111, no. 9, pp. 21732181, 2011.

[4] T. M. H. Eijsvogels, M. T. W. Veltmeijer, T. H. A. Schreuder, F. Poelkens, D. H. J. Thijssen, and M. T. E. Hopman, "The impact of obesity on physiological responses during prolonged exercise," International Journal of Obesity, vol. 35, no. 11, pp. 1404-1412, 2011.

[5] D. M. Savastano, A. M. Gorbach, H. S. Eden, S. M. Brady, J. C. Reynolds, and J. A. Yanovski, "Adiposity and human regional 
body temperature," American Journal of Clinical Nutrition, vol. 90, no. 5, pp. 1124-1131, 2009.

[6] I. B. Song, An Introduction to Sasang Constitutional Medicine, Jimoondang International, Seoul, Republic of Korea, 2005.

[7] J. Y. Kim and D. D. Pham, "Sasang constitutional medicine as a holistic tailored medicine," Evidence-Based Complementary and Alternative Medicine, vol. 6, supplement 1, pp. 11-19, 2009.

[8] D. D. Pham, S. Y. Cha, and J. Y. Kim, "Re-interpretation of traditional Asian medicine with constitutional perspective," Integrative Medicine Research, vol. 2, no. 1, pp. 1-6, 2013.

[9] K. Sohn, A. Jeong, M. Yoon, S. Lee, S. Hwang, and H. Chae, "Genetic characteristics of Sasang typology: a systematic review," Journal of Acupuncture \& Meridian Studies, vol. 5, no. 6, pp. 271-289, 2012.

[10] E. S. Jang, J. H. Do, H. Jin, K. Park, B. Ku, and S. Lee, "Predicting Sasang constitution using body-shape information," Evidence-Based Complementary and Alternative Medicine, vol. 2012, Article ID 398759, 6 pages, 2012.

[11] S. J. Lee, S. H. Park, and H. Chae, "Temperament profiles of Sasang typology in a child clinical sample," Integrative Medicine Research, vol. 1, no. 1, pp. 21-25, 2012.

[12] J. Y. Choi, Y. S. Lee, and S. S. Park, "The characteristics of perspiration according to Sasang constitution," Journal of Korean Oriental Medicine, vol. 23, no. 4, pp. 186-195, 2002.

[13] K. Kim, S. Y. Oh, J. C. Joo, E. S. Jang, and S. W. Lee, "Comparison of digestion, feces, sweat, and urination according to Sasang constitution in the 20s and 60s," Journal of Sasang Constitutional Medicine, vol. 22, no. 2, pp. 70-81, 2010.

[14] J.-H. Do, E. Jang, B. Ku, J.-S. Jang, H. Kim, and J. Y. Kim, "Development of an integrated Sasang constitution diagnosis method using face, body shape, voice, and questionnaire information," BMC Complementary and Alternative Medicine, vol. 12, article 85, 2012.

[15] H. Park, J. J. Chun, J. Kim, and K. Kim, "A study on clinical application of the QSCCll (Questionnaire for the Sasang Constitution Classification 11)," Journal of Sasang Constitutional Medicine, vol. 14, no. 2, pp. 35-44, 2002 (Korean).

[16] D. D. Pham, J. Lee, B. C. Ku, Y. Y. Kim, and J. Y. Kim, "Relation between body mass index and resting metabolic rate, cardiorespiratory fitness and insulin sensitivity in Sasang typology for young male persons: an observational study," European Journal of Integrative Medicine, vol. 4, no. 2, pp. e159e167, 2012.

[17] K. Birch, D. McLaren, and K. George, "Fundamentals of exercise physiology," in Sport and Exercise Physiology, pp. 1-10, BIOS Scientific Publishers, New York, NY, USA, 2005.

[18] M. N. Cramer, A. R. Bain, and O. Jay, "Local sweating on the forehead, but not forearm, is influenced by aerobic fitness independently of heat balance requirements during exercise," Experimental Physiology, vol. 97, no. 5, pp. 572-582, 2012.

[19] C. B. Scott, "Contribution of anaerobic energy expenditure to whole body thermogenesis," Nutrition and Metabolism, vol. 2, article 14, 2005.

[20] H. Momose, M. Sakaguchi, T. Nakazima, and T. Oohashi, "Development of flow control digital perspiration meter," IEICE Technical Report MBE2008-39, 2008, (Japanese).

[21] C. H. Y. Ling, A. J. M. de Craen, P. E. Slagboom et al., "Accuracy of direct segmental multi-frequency bioimpedance analysis in the assessment of total body and segmental body composition in middle-aged adult population," Clinical Nutrition, vol. 30, no. 5, pp. 610-615, 2011.
[22] D. Du Bois and E. F. Du Bois, "A formula to estimate the approximate surface area if height and weight be known," Nutrition, vol. 5, no. 5, pp. 303-311, 1989.

[23] PMCMR Package, http://cran.r-project.org/web/packages/ PMCMR/PMCMR.pdf.

[24] F. E. Marino, Z. Mbambo, E. Kortekaas et al., "Advantages of smaller body mass during distance running in warm, humid environments," Pflugers Archiv European Journal of Physiology, vol. 441, no. 2-3, pp. 359-367, 2000.

[25] S. C. Dennis and T. D. Noakes, "Advantages of a smaller bodymass in humans when distance-running in warm, humid conditions," European Journal of Applied Physiology and Occupational Physiology, vol. 79, no. 3, pp. 280-284, 1999.

[26] M. Yokota, L. G. Berglund, and G. P. Bathalon, "Female anthropometric variability and their effects on predicted thermoregulatory responses to work in the heat," International Journal of Biometeorology, vol. 56, no. 2, pp. 379-385, 2012.

[27] M. Yokota, G. P. Bathalon, and L. G. Berglund, "Assessment of male anthropometric trends and the effects on simulated heat stress responses," European Journal of Applied Physiology, vol. 104, no. 2, pp. 297-302, 2008.

[28] Y. Epstein, Y. Shapiro, and S. Brill, "Role of surface area-to-mass ratio and work efficiency in heat intolerance," Journal of Applied Physiology, vol. 54, no. 3, pp. 831-836, 1983.

[29] G. A. Selkirk and T. M. McLellan, "Influence of aerobic fitness and body fatness on tolerance to uncompensable heat stress," Journal of Applied Physiology, vol. 91, no. 5, pp. 2055-2063, 2001.

[30] D. D. Pham, J.-H. Do, B. Ku, H. J. Lee, H. Kim, and J. Y. Kim, "Body mass index and facial cues in Sasang typology for young and elderly persons," Evidence-Based Complementary and Alternative Medicine, vol. 2011, Article ID 749209, 9 pages, 2011.

[31] Y.-M. Kim, B. Ku, C. J. Jung et al., "Constitution-specific features of perspiration and skin visco-elasticity in SCM," BMC Complementary and Alternative Medicine, vol. 14, article 24, 2014.

[32] J.-B. Lee, T. Matsumoto, T. Othman, and M. Kosaka, "Suppression of the sweat gland sensitivity to acetylcholine applied iontophoretically in tropical Africans compared to temperate Japanese," Tropical Medicine, vol. 39, no. 3-4, pp. 111-121, 1998.

[33] J.-B. Lee, J.-S. Bae, T. Matsumoto, H.-M. Yang, and Y.-K. Min, "Tropical Malaysians and temperate Koreans exhibit significant differences in sweating sensitivity in response to iontophoretically administered acetylcholine," International Journal of Biometeorology, vol. 53, no. 2, pp. 149-157, 2009. 


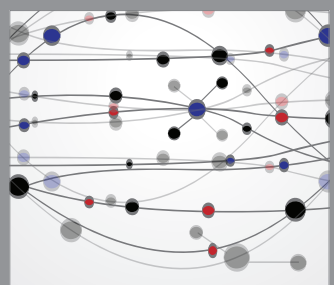

The Scientific World Journal
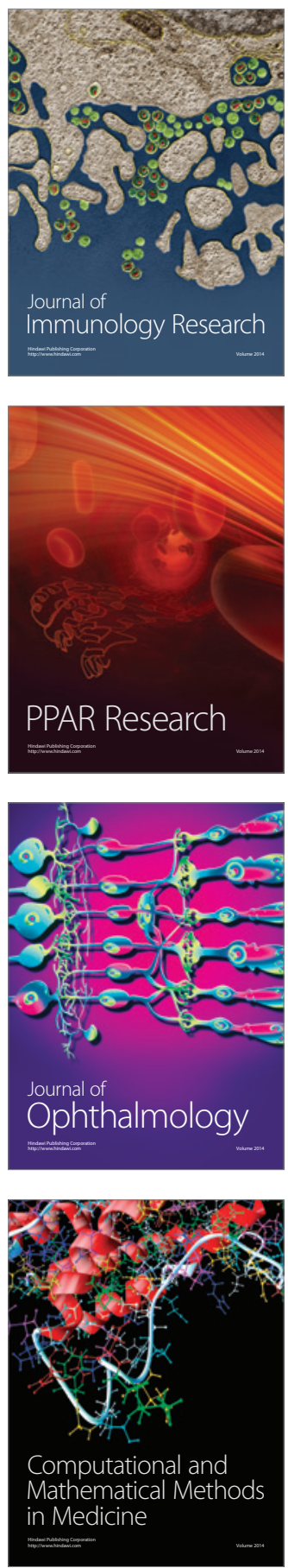

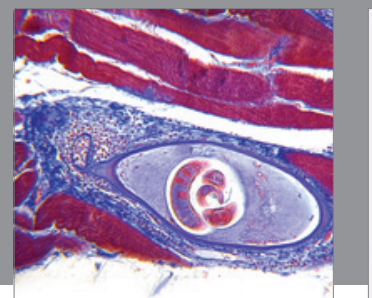

Gastroenterology

Research and Practice
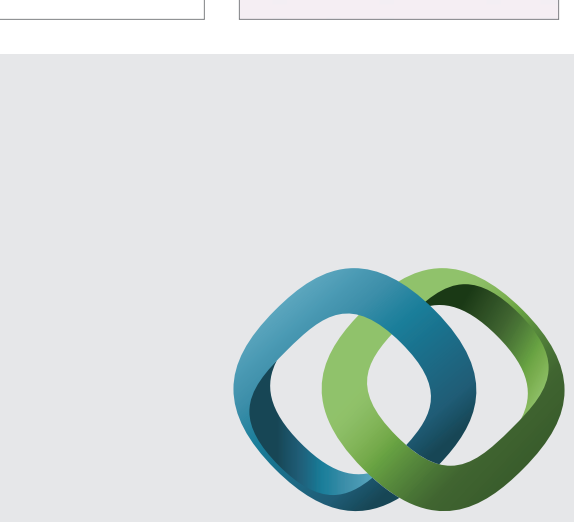

\section{Hindawi}

Submit your manuscripts at

http://www.hindawi.com
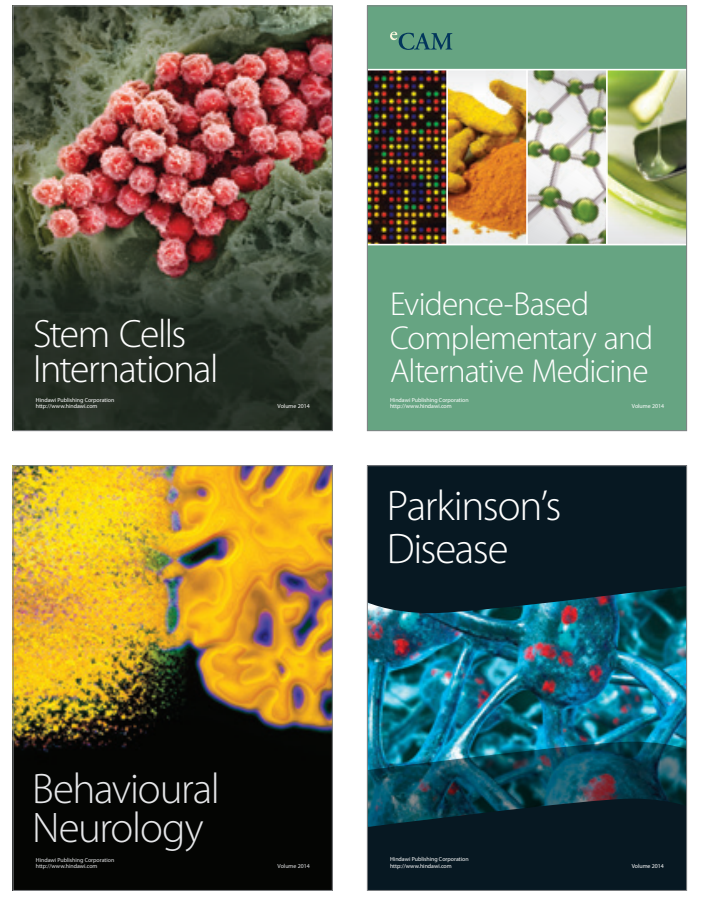
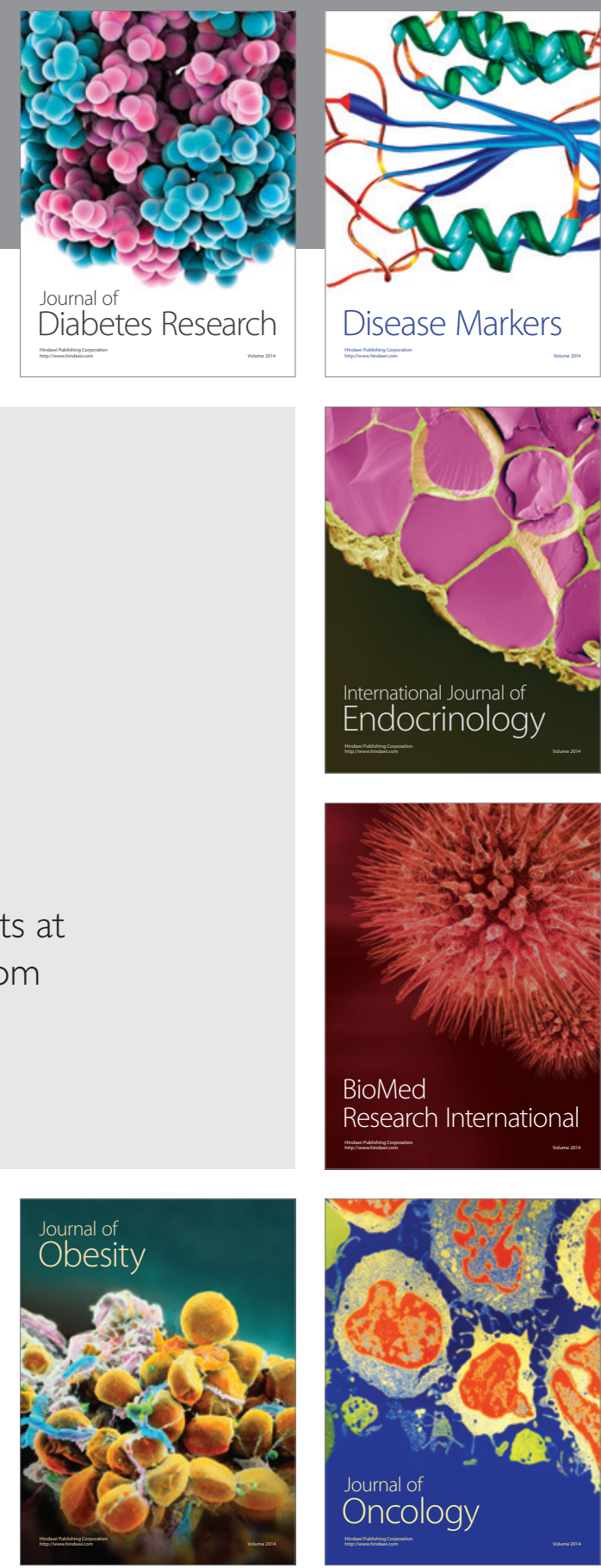

Disease Markers
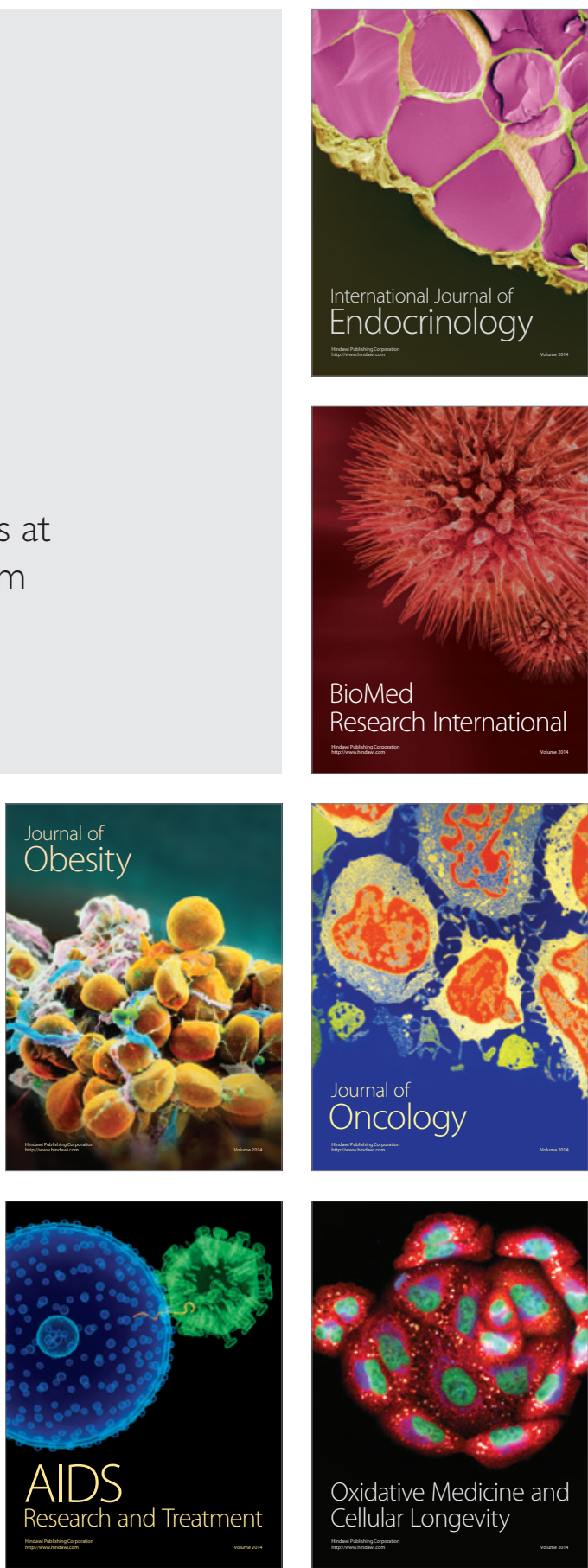